\title{
Bilateral Proximal Tibial Sleeve Fractures in a Child: A Case Report
}

\author{
Daniel Williams ${ }^{1,}$; Steven Kahane ${ }^{1}$; Daud Chou ${ }^{1}$; Krishna Vemulapalli ${ }^{1}$ \\ ${ }^{1}$ Department of Trauma and Orthopaedics, Queen's Hospital Romford, Rom Valley Way, Romford, Essex RM7 0AG, UK \\ ${ }^{*}$ Corresponding author: Daniel Williams, Department of Trauma and Orthopaedics, Queen's Hospital Romford, Rom Valley Way, Romford, Essex RM7 0AG, UK. Fax: +44-1689850915, \\ E-mail:Williams-dan@hotmail.co.uk \\ Received: February 15, 2015; Accepted: March 15, 2015
}

\begin{abstract}
Introduction: Asleeve fracture classically describes an avulsion of cartilage or periosteum with or without osseous fragments and usually occurs at the inferior margin of the patella. Tibial tubercle sleeve fractures in the skeletally immature are extremely rare.

Case Presentation:In this report the authors describe a 12-year-old boy with no systemic disease and no steroid use who sustained bilateral proximal tibial sleeve fractures whilst playing football. Both ruptures were associated with rupture of the medial patellofemoral ligament and tear of the medial retinaculum. Treatment was performed with primary end-to-end repair, reinforcement with bone anchors and cerclage wires with an excellent outcome.

Conclusions:We feel this rare, currently unclassified variant of a tibial tubercle avulsion fracture should be recognised and consideration taken to adding it to existing classification systems.
\end{abstract}

Keywords: Cartilage Fracture; Patella Tendon; Adolescent

\section{Introduction}

In children extensor mechanism ruptures are rare and usually occur through the substance of the patella tendon following a forceful contraction of the quadriceps muscle on a partially flexed knee. In 2002 Davidson first described and unusual injury pattern whereby a tendon is avulsed from the bone with a sleeve of cartilage or periosteum with or without osseous fragments (1). This can also occur from the inferior pole of the patella and this pattern of injury has been described (2-6). Patella tendon sleeve fractures often have subtle radiographic findings. A high level of clinical suspicion is essential to detect this injury. Bilateral ruptures of the extensor mechanism in preadolescence is extremely rare (7-9) and there is only one report of this occurring as a sleeve fracture in the literature (3).

This case study describes bilateral tibial tubercle sleeve avulsion fractures associated with a medial patellofemoral ligament (MPFL) rupture in a fit and well child with no systemic illness, no history of steroid use and no previous injury.

\section{Case Presentation}

We present a 12-year-old Afro-Caribbean boy who presented to the accident and emergency department unable to weight bear. He described playing football on grass when his left knee twisted. He felt his patella dislocate and fell to the ground on flexed knees with immediate pain and swelling in both knees.

His past medical history was unremarkable with no previous illnesses and no history of steroid use, previous dislocated joints or tendon ruptures.
Examination revealed swelling of both knees with global tenderness around the anterior aspect. He was unable to straight leg raise or hold the leg in extension when passively positioned. He had a normal Beighton score.

Radiographs performed in the Accident and Emergency department revealed a high riding and tilted patellae suggestive of a rupture. No osseous fragments were identified on plain radiographs and MRI was used to confirm the diagnosis of bilateral proximal tibial metaphyseal sleeve fractures (Figure 1).

Operative repair of the right patellar tendon ruptures was performed the following day. Intra-operative findings revealed the patellar tendon was avulsed from the tibial

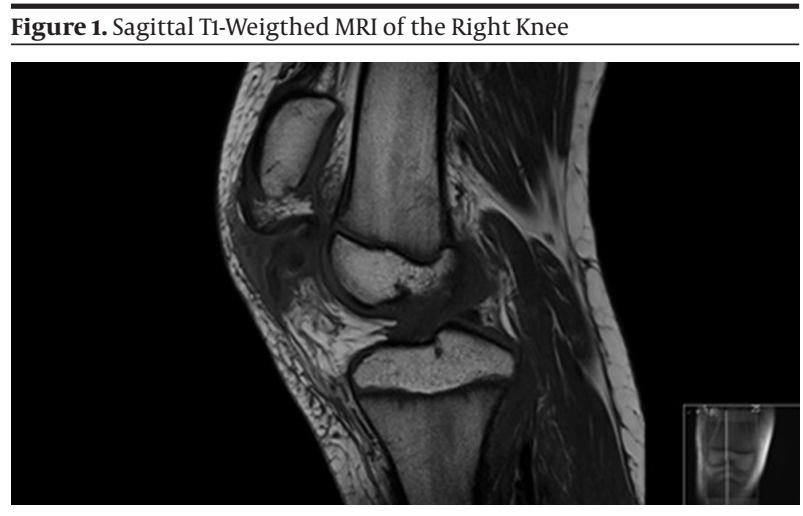

Demonstrating a complete avulsion of the patellar tendon from the tibia tuberosity with patella alta ( $4 \mathrm{~cm}$ retraction). There is an avulsion of two small fragments of bone. Signal change seen in the proximal and distal patellar tendon are suggestive of an underlying patellar tendinosis. Coronal MRI showed an extensive tear of the medial retinaculum and the MPFL.

Copyright (C) 2015, Kashan University of Medical Sciences. This is an open-access article distributed under the terms of the Creative Commons Attribution-NonCommercial 4.0 International License (http://creativecommons.org/licenses/by-nc/4.0/) which permits copy and redistribute the material just in noncommercial usages, provided the original work is properly cited. 


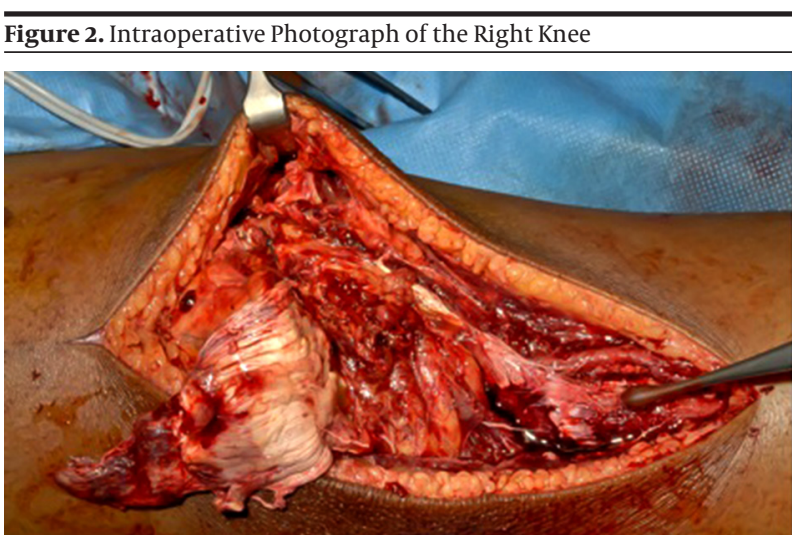

Through a midline incision the ruptured patella tendon has been reflected. The tibial tuberosity is marked with a curette demonstrating extensive periosteal stripping

tuberosity with a bony fragment and sleeve of periosteum (Figure 2). There was complete disruption of the retinaculum and there were no intra-articular injuries. The tendon was repaired using a four stranded Ethibond suture secured distally through two drill holes in the tibia. The bony avulsion fragment was reattached with suture anchors. The retinaculum was then repaired and then supplemented with box-configuration wiring. A good range of motion was achieved without any gapping of the tendon repair. The leg was immobilized in an above knee cylinder cast.

During the case the patients' temperature, $\mathrm{CO}_{2}$ and lactate increased. The patient was managed for malignant hyperpyrexia and the contralateral leg was not operated on during the same sitting. The patient was thoroughly investigated for malignant hyperpyrexia, connective tissue and metabolic disease. All investigations came back as negative. The contralateral leg was operated on using the same technique at a specialist center with appropriate anaesthetic support.

Post operatively the patient was mobilised as pain allowed with crutches in above knee cylinder casts for six weeks. At six weeks hinged knee braces were applied locked at 0 - 60 degrees for two weeks and then 0 - 90 degrees for two more weeks. He had a course of physiotherapy and a full range of movement and function was recovered at four months. The patient has no discomfort or irritation form the cerclage wires and as given the previous anaesthetic problems the decision was made to leave them in situ.

\section{Discussion}

In childhood, patella tendon rupture is rare and tends to occur at the osteotendinous interface. Considerable force equivalent to 17.5 times body weight is required to rupture a human adult patella tendon and as such is very rare in childhood (10). Bilateral patella tendon rupture in pre-adolescence is extremely rare with only three reported cases in the literature (7-9). The cases described by Moretti et al. (8) and Kim et al. (7) both describe sleeve type avulsion injuries from the inferior pole of the patellar. Muratli et al. (9) is the first to describe a bilateral sequential mid-substance tendon rupture in a nine-year old.

Recurrent microtrauma combined, genetic and metabolic susceptibility play a role in the development of tendinosis and predisposes individuals to a tendon rupture. In our case there was no significant systemic disease or steroid use that would otherwise predispose to tendon rupture.

Patellar tendinopathy or Jumpers knee is common in adolescent athletes and usually starts at distal pole of the patella near the insertion of the patella tendon. Rupture in these individuals however remains low. Unlike the other case of tibial tuberosity avulsion fractures $(1,3,6)$ in this case the MRI scan confirmed severe patellar tendinosis affecting the whole of the patellar tendon. Repetitive microtrauma may well have been a predisposing factor in this case.

The diagnosis of bilateral patella tendon rupture and sleeve fractures can be difficult given its rarity, lack of osseous fragments on plain radiographs and the symmetry of the examination findings. With accurate diagnosis and prompt repair excellent outcomes are described.

Patella tendon repair is dependent on location and tissue quality with direct end to end repair, suture anchors and cerclage wires all commonly used. Some authors use allograft including Muratli et al. (9) who used Achilles tendon allograft in the nine-year old patient to reinforce the bilateral mid-substance repair with excellent results. The senior author would recommend suture anchors to augment the repair in this variant of tibial tuberosity sleeve fractures.

The tibial tuberosity sleeve fracture is a rare variant of the tibial tuberosity avulsion fracture originally classified by Watson-Jones (11) and Ogden (12). Ryu and Debenham (13) modified the Watson-Jones classification to include a tibial tuberosity fracture that extends into the posterior cortex (Type IV). We would support a classification system to include a tibial tuberosity sleeve fracture either as a type IV injury in a modified Ogden classification (Table 1) or as a Type V injury in a further modification of the Watson-Jones system.

Table 1. A Modification of Ogdens 1980 Classification of Tibial Tubercle Fractures to Include a Suggested New Type IV Injury; the Tibial Tuberosity Sleeve Fracture

\begin{tabular}{lcc}
\hline & Type A & Type B \\
\hline Type I & A simple avulsion of the ossification centre & IA + Displacement \\
Type II & Fracture-separation of the anterior tibial tuberosity & IIA + Comminution \\
Type III & Fracture extending into the knee & IIIA + Comminution \\
Type IV & Tibial tuberosity sleeve fracture & IV + Osseous fragmentation \\
\hline
\end{tabular}


Bilateral concurrent tibial tuberosity avulsion fractures are extremely rare in pre-adolescent children. All paediatric orthopaedic trauma surgeons should be aware of this rare variant of the sleeve avulsion fracture and that an excellent outcome is achievable with surgical repair.

\section{References}

1. Davidson D, Letts M. Partial sleeve fractures of the tibia in children: an unusual fracture pattern. J Pediatr Orthop. 2002;22(1):36-40.

2. Bates DG, Hresko MT, Jaramillo D. Patellar sleeve fracture: demonstration with MR imaging. Radiology. 1994;193(3):825-7.

3. Desai RR, Parikh SN. Bilateral tibial tubercle sleeve fractures in a skeletally immature patient. Case Rep Orthop. 2013;2013:969405.

4. Gao GX, Mahadev A, Lee EH. Sleeve fracture of the patella in children. J Orthop Surg. 2008;16(1):43-6.

5. Houghton GR, Ackroyd CE. Sleeve fractures of the patella in children: a report of three cases.J Bone Joint Surg Br.1979;61-B(2):165-8.
6. Kosuge DD, Balaji VB, Ahad N, Vemulapalli K. Proximal Tibial Sleeve Fracture: Case Report of a Rare Injury and Review of the Literature. Eur J Trauma Emerg Surg. 2009;36(4):388-91.

7. Kim JR, Park H, Roh SG, Shin SJ. Concurrent bilateral patellar tendon rupture in a preadolescent athlete: a case report and review of the literature.J Pediatr Orthop B. 2010;19(6):511-4.

8. Moretti B, Notarnicola A, Moretti L, Garofalo R, Patella V. Spontaneous bilateral patellar tendon rupture: a case report and review of the literature. Chir Organi Mov. 2008;91(1):51-5.

9. Muratli HH, Celebi L, Hapa O, Bicimoglu A. Bilateral patellar tendon rupture in a child: a case report. Knee Surg Sports Traumatol Arthrosc. 2005;13(8):677-82.

10. Zernicke RF, Garhammer J, Jobe FW. Human patellar-tendon rupture. J Bone Joint Surg Am. 1977;59(2):179-83.

11. Watson-Jones R. Fractures and Joint Injuries. 5th edition. Vol. 2. Baltimore, Md, USA: Williams \& Wilkins; 1955.

12. Ogden JA, Tross RB, Murphy MJ. Fractures of the tibial tuberosity in adolescents. J Bone Joint Surg Am. 1980;62(2):205-15.

13. Ryu RKN, Debenham JO. An unusual avulsion fracture of the proximal tibial epiphysis. Clin Orthop 1985.194:181-4. 